\title{
MOOCS: A New Way of Teaching Transmission Electron Microscopy
}

\author{
Cécile Hébert ${ }^{1,2^{*}}$ and Duncan T.L. Alexander ${ }^{1}$
}

1. Electron Microscopy and Spectroscopy Laboratory (LSME), Institute of Physics (IPHYS), Ecole Polytechnique Fédérale de Lausanne (EPFL), Lausanne, Switzerland.

2. Institute of Materials (IMX), Ecole Polytechnique Fédérale de Lausanne, Lausanne, Switzerland.

* Corresponding author: cecile.hebert@epfl.ch

Transmission Electron Microscopy (TEM) is a very important tool in many domains of materials science and physics, hence many universities with TEM groups offer lectures at the master or doctoral level. Such courses are however often embedded into a given curriculum and offered on a yearly basis. This is not always in line with the need for training of new TEM users.

Very good textbooks exist, e.g. [1-3], but operating a TEM is a dynamic process, where many things are much easier to understand by watching an image change as one parameter is continuously adjusted. For example, seeing a diffraction pattern evolving as a sample is tilted from zone axis to a systematic row condition, or watching the "structure" in an amorphous carbon film change as defocus is applied in the presence of astigmatism, is not the same as having a series of static images in a book. TEM lectures can instead be complemented by demonstrations at the microscope where the operator shows the application of principles that were explained before, but this is not done in the same room and requires investment of operator and instrument time.

A modern TEM instrument offers an incredible variety of techniques and, in a university where users come to TEM from different fields (materials, physics, chemistry, nanotechnology, environment, ...), individual users will need different techniques. Some will need high resolution TEM, some will need scanning TEM, some will need selected area diffraction or dark field imaging, etc. Therefore, it is of great advantage if users can access modular lectures tailored to their needs. Further to this, the heterogeneity of backgrounds for these different research fields means that there can be significant differences in understanding of TEM theory from researchers who need to use TEM. As we have seen regularly at EPFL, PhD students and postdocs who want to use TEM from domains such as chemistry may have had little or no exposure to TEM theory during their studies.

The recent development of so-called MOOCS (Massive Open Online Courses) offers a very interesting possibility for the teaching of transmission electron microscopy to address these challenges:

- It allows the development of a set of dynamic and attractive lectures in the form of videos mixing conventional blackboard-like drawing with extracts from recordings made at the microscope.

- Concepts can be explained dynamically, with direct links to what happens at the microscope.

- Students can follow the lectures at their own pace, and can begin the course when compatible to their needs.

These considerations motivated us to develop an 8-week long online lecture course on TEM for materials science, which we have published on the Coursera platform [4]. Four weeks after launch, which took place $15^{\text {th }}$ of January, 2019, we had 684 enrolled students and over 300 active learners. The vast majority hold a master or bachelor degree, so they are most probably $\mathrm{PhD}$ or master students (Figure 1). Since the course is offered, "on-demand" on Coursera, students can register whenever they 
want. The timing of this conference 6 months after the launch of the MOOC will allow us to provide a first evaluation on its progress. We will report on the number of students, their success rate, origin (by countries and background), and general feedback.

As well as being offered as a standalone course on TEM theory accessible by an international audience somewhat akin to a text book usage - at the local level our online course can also be used to implement some of the latest innovations in teaching. As an example, in our master course on TEM at the EPFL we are using the online material to "flip the classroom". For 8 out of the 13 weeks of the course, the students are asked to follow the videos prior to class. Time usually devoted to in-person teaching is leveraged for interactive discussions and exercise sessions, and for having the students experiment and investigate the concept of the week in small groups at the microscopes. First trials of this approach in the last academic year indicated a positive outcome in terms of student feedback and exam results.

In this presentation, we shall also discuss the challenges and rewards that we faced in creating the MOOC. On the one hand, the TEM subject is highly theoretical, and the MOOC environment required that we purpose-build extensive material such as schematic diagrams and appropriate exercises from the ground up. On the other hand, "simple" conceptual experiments often proved surprisingly challenging to realize as screenable demonstrations recorded at the microscope. Finally, we shall describe our plans for the future, where we aim to create new modules covering other core aspects of transmission electron microscopy [5].

\section{References:}

[1] D.B. Williams and C.B. Carter, "Transmission Electron Microscopy: A Textbook for Materials Science" (Springer, New York).

[2] L. Reimer and H. Kohl, "Transmission Electron Microscopy: Physics of Image Formation" (Springer, New York).

[3] M. De Graef, "Introduction to Conventional Transmission Electron Microscopy" (Cambridge

University Press, Cambridge).

[4] https://www.coursera.org/learn/microscopy

[5] The authors acknowledge funding from the EPFL as well as the support of the team at the EPFL

MOOC Factory, and in particular the incredible work done by Matthew Goodman on the video editing.
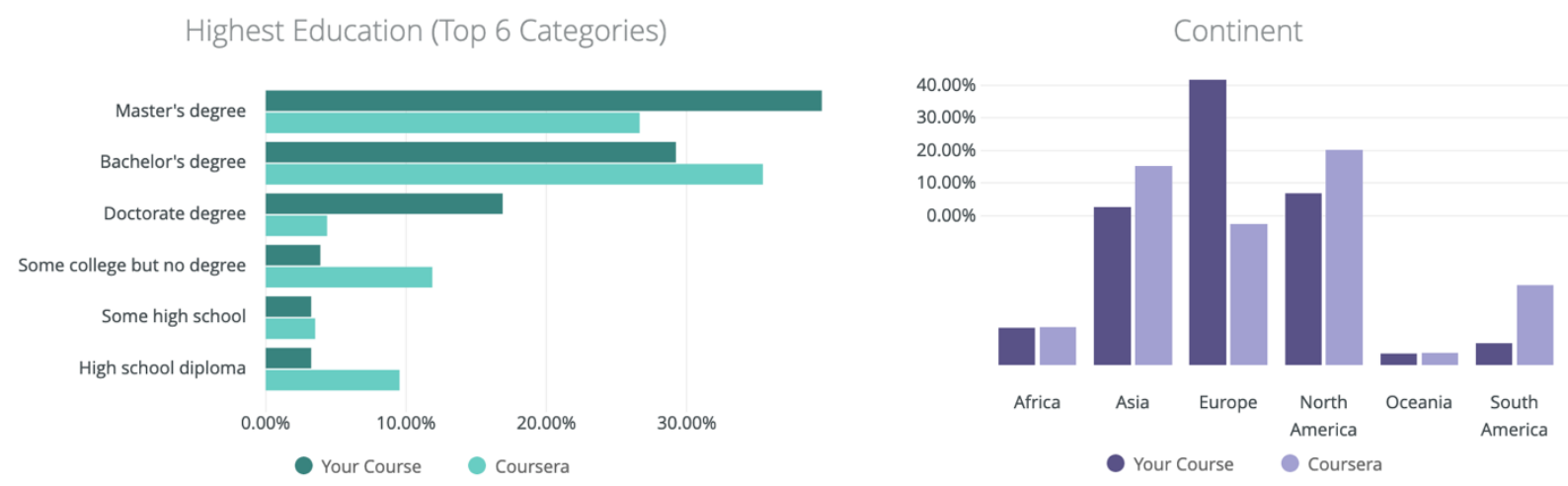

Figure 1. Highest degree held by the registered students, and continent of origin. 